\title{
Energy cost assessment of a polar based controller applied to a flexible rotor supported by AMB
}

\author{
Benjamin DEFOY*, Thomas ALBAN* and Jarir MAHFOUD** \\ * GE Oil\&Gas Thermodyn \\ 480 allée G. Eiffel, BP 119, 71203 Le Creusot Cedex, France \\ ** Université de Lyon, INSA-Lyon, LaMCoS UMR5259 \\ 18-20 rue des Sciences, Bât J. d'Alembert, 69621 Villeurbanne Cedex, France \\ E-mail: jarir.mahfoud@insa-lyon.fr
}

\section{Received 16 February 2015}

\begin{abstract}
From a designer point of view, optimizing the energy necessary for the control is an important element that could lead to downsize the control cabinet and to an increase of margins according to power amplifier capabilities. Also, reducing the energy used, generates a less significant environmental impact. The aim of this work was to develop and to assess a new approach using polar coordinates to observe and actuate the control of a flexible rotor supported by Active Magnetic Bearings (AMBs). Two fuzzy logic based controllers used to modulate the actuating forces were developed and assessed. The first is a SISO fuzzy PID controller for which the input is the displacement measured along an action line while the output is the force applied in the same direction. The second controller considers each bearing as a single MIMO system with the displacements in the two orthogonal directions as inputs, by managing two significant physical quantities, namely tangential and radial speeds. The "mechanical" performances are compared to those of an augmented PID controller. Then, the energy necessary for the control is compared. The study is first performed numerically and then validated experimentally. The results obtained show that the polar fuzzy controller presents the most suitable mechanical performances and energy costs
\end{abstract}

Key words : AMB, Active control, Flexible rotor, Polar coordinates, Fuzzy logic, Experiments

\section{Introduction}

Active Magnetic Bearings (AMB) are widely utilized in different industrial applications. Several applications have been successfully implemented in the field of turbomachinery (Maslen, 2008, Swann, et al., 2008) and several studies have been devoted to the formulation of new controllers that enable better design and performance in operating situations, with acceptable levels of stability and robustness (Schweitzer and Maslen, 2009, Sahinkaya, et al., 2010, Fitro and knospe, 2002 and Lei and Palazzolo, 2008). The most successful controller for industrial applications is the augmented PID controller, since it permits designers to master the control process. However, the control of flexible rotors remains difficult due to unavoidable spillover effects.

Several studies have been devoted to the formulation of new controllers that enable better design and performance in operating situations, with acceptable levels of stability and robustness (Couzon and Der Hagopian, 2007, Chen, et al., 2009, Font, et al., 1997). The most successful of these controllers is the $\mu$-synthesis controller whose parameters can be tuned automatically. Nevertheless, the optimization process is delicate as the number of specifications is greater than the number of parameters available. Consequently, the augmented PID controller is still widely used.

Industrial requirements involve several constraints regarding performance, robustness, ease of implementation and final tuning. These requirements are dictated by international standards such as ISO 14839-1 (2002), ISO 14839-2 (2004) and ISO 14839-3 (2006) and API 617 (2002). The specifications of the final users must also be taken into account. Nowadays, the spectrum of applications covered by AMBs is widely diversified. Each application has its own operating conditions and excitations. 
Previous studies have shown that fuzzy controller approaches are well adapted for controlling flexible structures (Mahfoud and Der Hagopian, 2011, Mahlis, et al., 2005). Moreover, the advantage of fuzzy control is that it can be used in complex systems such as nonlinear and time-variant systems and those including uncertainties (Borne, 1998). In addition, fuzzy controllers are less sensitive to variations of system parameters and they allow the utilization of membership functions adapted to the dynamic behavior of the system considered. They also enable the calculation of nuanced actions and take into account several data variations, thereby ensuring robust behavior (Fuh and Tung, 1997, Golob and Tovornik, 2003, Qiao and Mizumoto, 1996). Defoy et al., (2014) developed a new control strategy that utilizes polar coordinates. The originality of the methodology developed is that it manages two significant physical quantities, namely tangential and radial velocities, which are associated with steady state and transient behaviors, respectively. The outputs are the forces computed in the polar coordinates and converted into currents that drive the action lines at the same time.

The work is part of a research program to develop new control approaches dedicated to turbomachinery. In this paper the mechanical performances and also the energy necessary for the control of an academic test rig were assessed experimentally. Two fuzzy logic based controllers are developed and assessed in terms of "mechanical performances" and energy consumption. The aim is to evaluate the possibility of optimizing energy consumption to reduce the size of the control cabinet and increase margins of maneuver according to power amplifier capacities. In addition, energy reduction will be expressed by less significant environmental impact, of particular concern at present. The paper is divided into several sections. After a brief introduction, the experimental apparatus is described, after which the control strategies are presented. The numerical model of the test rig used for the numerical implementation is set out in order to describe and to adjust the design approach applied. The experimental results obtained are then presented. Finally, the advantages and disadvantages are highlighted and the conclusions are summarized in the final section.

\section{Experimental setup}

The experiments were performed using an academic test rig (Fig. 1). It is a commercial product manufactured by SKF® and was delivered with a dedicated PID controller. The test rig is equipped with two identical AMBs called NDE (Non Drive End) and DE (Drive End) bearings. Each bearing has a maximum static capacity of $280 \mathrm{~N}$. The action lines are positioned in the configuration load between the axes. They are powered in differential driving mode with a bias current of 1A. Currents are provided in the range of 0-3A using PWM amplifiers. Two displacement sensors (variable reluctance probes) are integrated in the housing of each bearing and are non-colocalised with the actuators. The Input/Output panel gives access to the displacements measured and enables entering current settings for the amplifiers. Each AMB has one back-up bearing with a clearance radius of $0.1 \mathrm{~mm}$.

The shaft is composed of three parts bolted together. A central part (diameter: $19.05 \mathrm{~mm}$; length $344 \mathrm{~mm}$ ) with a disc $120 \mathrm{~mm}$ in diameter and $25 \mathrm{~mm}$ long is placed at mid-span, together with two shaft ends ( $35 \mathrm{~mm}$ of the main diameter). The stack of laminated steel sheets is shrunk on each of these two shafts. Due to the different lengths of the latter (190.5mm at DE and $110.5 \mathrm{~mm}$ at NDE), the rotor is not symmetric in relation to the central disk. The total rotor length is $645 \mathrm{~mm}$. The rotor mass is $5.89 \mathrm{~kg}$. Three balancing planes are available: one at the central disc and one at each bolted plane. The rotor is driven by a 500W electric motor with a maximum speed of 12,600rpm. Power transmission is provided by a flexible coupling. The operating speed range used in this work is from 0 to $10,000 \mathrm{rpm}$, which includes three critical speeds (two forward rigid modes and the first forward flexible mode). The speed of the rotor is monitored by using a speed sensor placed close to the motor.

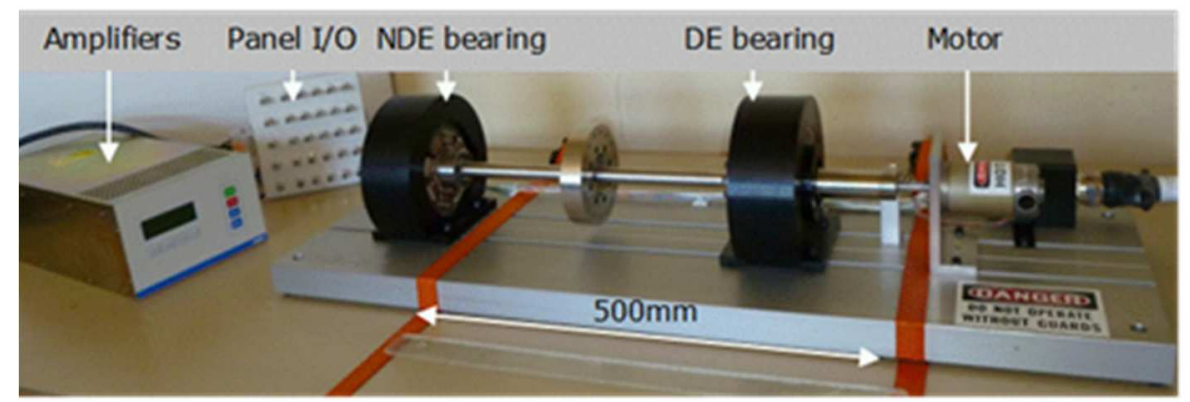

Fig. 1 Experimental test rig 
The frequency bandwidth considered in this study is $0-2 \mathrm{kHz}$. The test rig can be controlled either by using the dedicated device or externally via the input/output panel. The new controller is implemented under Simulink® and dSpace ${ }^{\circledR}$ environments, with a sampling frequency of $20 \mathrm{kHz}$.

\section{Numerical model}

Two fuzzy controllers were developed with numerical simulations and then applied to the academic test rig. The numerical model was necessary in order to establish the design methodology and tune the controller parameters. The numerical model of the rotor was formulated with the finite element method, without experimental identification. The detailed model of the rotor and the mode shapes are presented in Fig. 2. For the sake of clarity, only the shapes of the first three flexible modes are plotted.

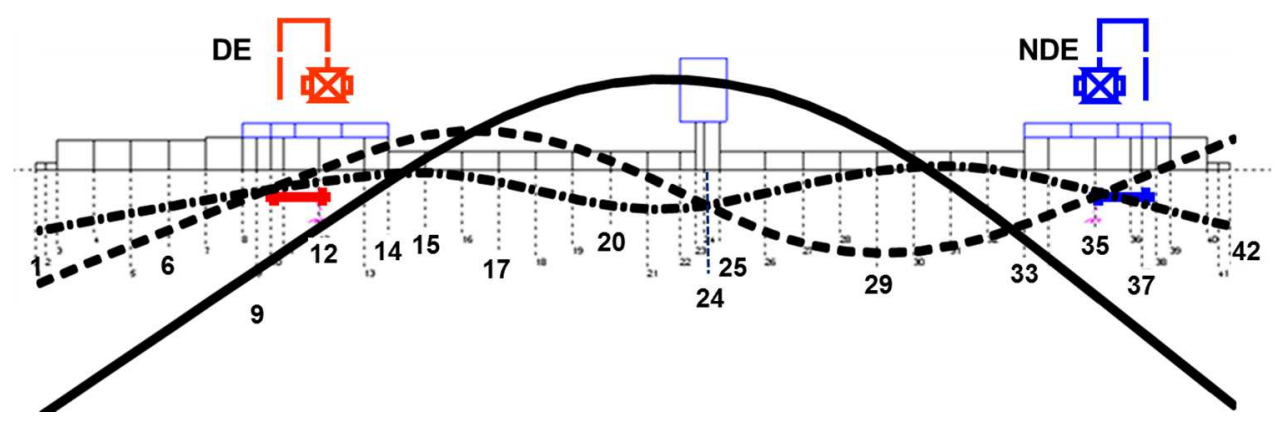

Fig. 2 Detailed model of the rotor and mode shape of the first three modes

The rotor model is composed of the following elements: rigid discs, flexible shafts and bearings. Regarding the shaft, it is modelled with 41 Timoshenko beam elements with two nodes and 4 degrees of freedom, namely two displacements and two rotations per node.

The dynamic behavior of the rotor studied can be expressed by the following differential equation (Lalanne and Ferraris, 1998)

$$
[M] \ddot{\delta}+\left[C+\dot{\phi} C_{g}\right] \dot{\delta}+\left[K+\ddot{\phi} K_{g}\right] \delta=F_{e x t}+F_{A M B}
$$

where $[M]$ is the mass matrix, $[K]$ is the stiffness matrix, $[C]$ is the damping matrix, $\left[K_{g}\right]$ and $\left[C_{g}\right]$ represent the gyroscopic contribution, $\delta$ is the vector of the generalized displacements, and $\dot{\varnothing}$ is the angular velocity. On the right hand, we grouped the influence of the external forces $\left(F_{\text {ext }}\right)$ that include disturbance forces $F_{\text {disturbances }}$ and unbalance forces $F_{b}$, the contribution of the seals $\left(F_{\text {seals }}\right)$ and the contribution of the magnetic bearings $\left(F_{A M B}\right)$. Under steady state conditions, or for small speed variation, the angular acceleration $\ddot{\emptyset}$ can be neglected. Consequently, Eq. (1) is rewritten as:

$$
[M] \ddot{\delta}+\left[C+\dot{\phi}\left[C_{g}\right]\right] \dot{\delta}+[K] \delta=F_{e x t}+F_{A M B}
$$

The state space system presentation is more convenient for control purposes, especially in the case of multi-inputs. The equations of motion become:

$$
\left[\begin{array}{c}
\dot{\delta} \\
\ddot{\delta}
\end{array}\right]=\left[\begin{array}{cc}
{[0]} & {[I]} \\
-[M]^{-1}[K] & -[M]^{-1}[C]-[M]^{-1}\left[C_{g}\right] \dot{\phi}
\end{array}\right]\left[\begin{array}{l}
\delta \\
\dot{\delta}
\end{array}\right]+\left[\begin{array}{c}
{[0]} \\
{[M]^{-1}}
\end{array}\right] F
$$

where $F$ is the sum of all second member contributions. For practical reasons, the dynamic matrix can be split into a speed dependent part that expresses the gyroscopic effects, and a speed non-dependent part. In this way, the dynamic matrix will express the contribution of the linear system only, while the nonlinear contribution is considered as restoring forces: 


$$
\left[\begin{array}{c}
\dot{\delta} \\
\ddot{\delta}
\end{array}\right]=\left[\begin{array}{cc}
{[0]} & {[I]} \\
-[M]^{-1}[K] & -[M]^{-1}[C]
\end{array}\right]\left[\begin{array}{l}
\delta \\
\dot{\delta}
\end{array}\right]+\left[\begin{array}{c}
{[0]} \\
{[M]^{-1}}
\end{array}\right]\left(-\dot{\phi}\left[C_{g}\right] \dot{\delta}+F\right)
$$

In order to reduce the calculation time, an important criterion for real-time control, the modal reduction method is used to keep only the first 14 modes (to conform to the bandwidth frequency).

The calculations of the natural frequencies and the mode shapes were not performed with AMBs but only with direct stiffness (no cross stiffness and no damping). The direct stiffness value $\left(8.105 \mathrm{Nm}^{-1}\right)$ was chosen in order to be as close as possible of the characteristics of the magnetic bearings. The designer is free to determine the AMB stiffness. Typically, this direct stiffness value is chosen where the slope of the three first modes varies as a function of the critical speed map. This value enables significant action on the system strain energy and damping. This constant stiffness is then removed and the AMB is considered as a restoring force. ROTORINSA® software is used to compute the modal matrixes. Table 1 presents the natural frequencies of the rotor computed for the frequency bandwidth studied.

Table 1: Rotor natural frequencies

\begin{tabular}{cccc}
\hline \hline Modes & $\begin{array}{c}\text { Orpm } \\
(\mathrm{Hz})\end{array}$ & $\begin{array}{c}15000 \mathrm{rpm} \\
\text { Backward }(\mathrm{Hz})\end{array}$ & $\begin{array}{c}\text { 15000rpm } \\
\text { Forward }(\mathrm{Hz})\end{array}$ \\
\hline Rigid 1 & 70 & 69 & 70 \\
Rigid 2 & 90 & 88 & 92 \\
1 & 134 & 130 & 138 \\
2 & 413 & 345 & 476 \\
3 & 766 & 719 & 822 \\
4 & 1139 & 1043 & 1287 \\
5 & 2643 & 2627 & 2663 \\
\hline
\end{tabular}

The general modeling approach is presented in Fig. 3. The dynamic matrix expresses only the linear contribution of the system studied. All the other contributions, i.e. frequency, speed and time dependent physical quantities such as gyroscopic effects, AMB forces and the seal effects, are considered as restoring forces. Consequently, the mode shapes can be calculated easily as the Eigen vectors of the linear dynamic matrix.

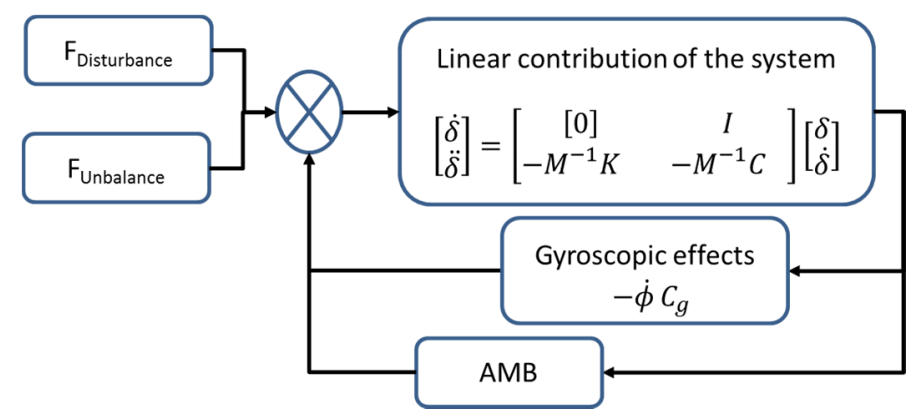

Fig. 3 General modeling approach

The AMB is powered in differential driving mode with a bias current $I_{0}$ and a control current $i$ (5).

$$
F_{B}=4 \xi_{B} \mu_{0} S N^{2}\left[\frac{\left(I_{0}-i\right)^{2}}{\left(2 g_{0}-2 x+\frac{L}{\mu_{r}}\right)^{2}}-\frac{\left(I_{0}+i\right)^{2}}{\left(2 g_{0}+2 x+\frac{L}{\mu_{r}}\right)^{2}}\right]
$$

where $\xi_{B}$ is a coefficient dependent on the bearing geometry ( 0.92 in our case), $\mu_{0}$ the permeability of vacuum space, $S$ the pole area, $N$ the number of turn in one coil, $g_{0}$ the nominal air gap, $x$ the rotor position, $\mu_{r}$ the relative permeability of the iron core, and $L$ the average length of magnetic flux lines. 
Using a bias current considerably reduces the non-linearity of the magnetic actuator, leading to almost linear behavior in the range of nominal displacement. In order to simplify the study, the AMB forces (Eq. 5) are linearized around the operating point with respect to the bearing geometry (6).

$$
K_{i}=\left.\frac{\partial F_{B}}{\partial i}\right|_{\substack{x=0 \\ i=I_{W}}}=\frac{-16 \xi_{B} \mu_{0} S N^{2} I_{0}}{\left(2 g_{0}+\frac{L}{\mu_{r}}\right)^{2}} ; K_{x}=-\left.\frac{\partial F_{B}}{\partial x}\right|_{\substack{x=0 \\ i=I_{W}}}=\frac{-32 \xi_{B} \mu_{0} S N^{2}\left(I_{0}^{2}+I_{W}^{2}\right)}{\left(2 g_{0}+\frac{L}{\mu_{r}}\right)^{3}}
$$

Hence the AMB is introduced in the model via the so-called current stiffness $K_{i}$ of $-97 \mathrm{NA}^{-1}$ and a negative stiffness $K_{x}$ of $-0.34 \mathrm{~N}_{\mu \mathrm{m}^{-1}}(7)$. The negative stiffness is a consequence of the attractive forces produced by the electromagnets when they are supplied with constant current. These attractive forces are inversely proportional to the squared value of the gap distance, and introduce the softening effects on the dynamic behavior.

$$
F_{B} \cong K_{i} i-K_{x} x
$$

On the other hand, the electronic part of AMB is represented with a first order low pass filter with a cut off frequency of $2 \mathrm{kHz}$. This transfer function takes into account the displacement probe bandwidth, the time delay introduced by digital signal processor and the amplifier response.

\section{Control strategies}

Three control strategies were assessed. The first is that developed by the test rig constructor, a fuzzy controller and a polar fuzzy controller. A simple PID was necessary for the Fuzzy based controllers. The SPID enabled the introduction of suitable stiffness and damping for low frequency, and the Fuzzy process was used to modulate the gains of the SPID. The general structure of the three controllers is presented in Fig. 4.

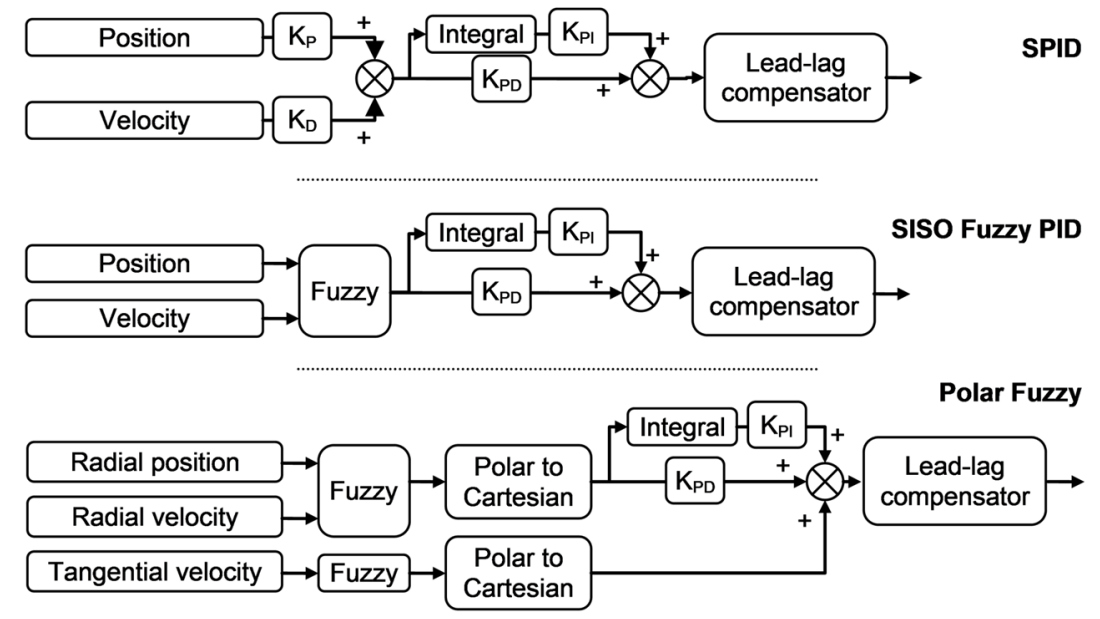

Fig. 4 Structure of the three controllers assessed

\subsection{SPID}

The first step of the fuzzy approach was the definition of the simple PID coefficients whose voluntarily simple characteristics were in the low frequency range. Usually, the characteristics are determined as a function of the dynamic behavior and the number of modes included in the operating conditions. In order to generalize the design process, the coefficients were chosen such that the damping was almost constant and with a low constant slope for the stiffness, for the operating frequency range considered (Fig. 5). Consequently, the controller became less efficient but more multifunctional. Figure 5 shows the variations of bearing stiffness and damping as a function of frequency. The evolutions of the natural frequency of the first four modes are also presented. It is noteworthy that the gyroscopic effect was almost negligible for the first three modes. 

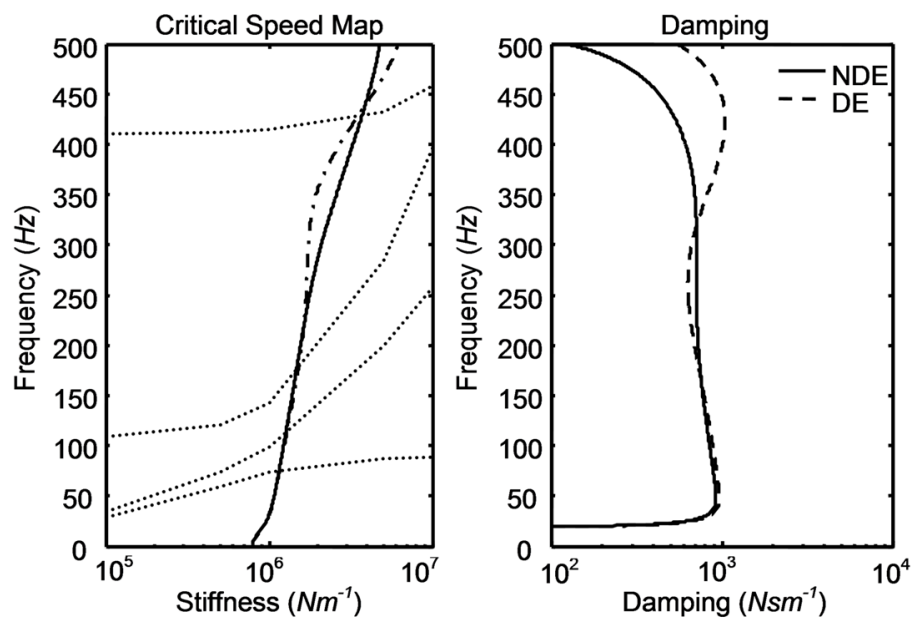

Fig. 5 Standardized stiffness and damping for the operating frequency range considered

\subsection{Fuzzy controller}

This consisted of the sum of a fuzzy PI with a fuzzy PD controller. The displacement was measured and the velocity calculated by numerical derivation. These two data (displacement and velocity) were used as inputs for the fuzzy controller. Four generalized bell shape membership functions were utilized (Matlab). These membership functions were associated with four fuzzy sets: positive/negative displacements and positive/negative velocities

The design of a membership function takes into account the measurement noise. The displacements and velocities are measured, and their respective amplitudes make it possible to obtain the measurement uncertainty directly. These amplitudes of displacements and velocities should be insufficient to change the prevalent membership function. On the other hand, in order to be efficient small amplitudes of displacement and velocity should belong wholly to one of the two fuzzy sets (positive or negative). Finally, as nominal behavior is considered and, the displacements and velocities are relatively small compared to the measurement noise, only two membership functions were utilized for each input of the fuzzy controller.

The rules were chosen in order to optimize the energy dissipation and minimize the kinetic energy of the rotor (the force sign is always opposite that of the velocity). The rules selected were:

1) When position and velocity have the same sign, the proportional gain is activated. Note that the other gains are always activated.

2) When the displacement and velocity have opposite signs, the damping gain is conserved but the stiffness is set to zero.

This study considers only the case of nominal behavior with low displacements. Consequently, if the membership functions are tuned too smoothly, a linear controller can approximate the non-linear one. And, if the membership functions are tuned with overly sharp transitions, disturbances can appear, leading to system instability. In our case, in which the ratio of tolerated displacements over the uncertainties due to measurements is small, there is a limited range of variation for the membership functions. This provides an interesting advantage for the fuzzy controllers without disturbing the system.

\subsection{Polar fuzzy controller}

Generally, the response of the system in acceleration or displacement is measured by using sensors placed along the structure. Regarding lateral behavior, two sensors oriented in two perpendicular axes are sufficient to describe the dynamic behavior. Then, the measured signals are processed and analyzed in time, frequency or time-frequency domains. We assume that this approach is quite suitable for non-rotating structures, but when dealing with rotating machinery there is a lack of information on rotational speed. This lack can be compensated by describing the response measured in the polar coordinates. Observation of the measurements in the polar domain can lead to easier interpretation of the dynamic behavior, where the steady state and the transient behaviors can be distinguished directly, particularly in the case of realtime controlled systems.

The transformation from Cartesian to polar representation is obtained classically as indicated in (8). Where $\mathrm{x}$ and $\mathrm{y}$ are the measured displacements along directions $\mathrm{x}$ and $\mathrm{y}$ in the Cartesian representation, while $\mathrm{r}$ and $\theta$ are the corresponding 
polar quantities.

$$
\left\{\begin{array}{c}
r=\sqrt{x^{2}+y^{2}} \\
\tan \theta=\frac{y}{x}
\end{array}\right.
$$

Then the radial and the tangential velocities, $V_{r}$ and $V_{t}$ respectively, are calculated as follows:

$$
\begin{gathered}
V_{r}=\dot{r} \\
V_{t}=r \dot{\theta}
\end{gathered}
$$

where $\dot{r}$ is obtained by numerical differentiation of the radial position.

In a steady state case and in the presence of synchronous excitation as unbalance, the orbit is circular (symmetric rotor), and the radial displacement is constant, thus the radial velocity is nil while the tangential velocity is constant. It is noteworthy that in the case of system dissymmetry, the orbit of displacement is elliptical and the radial speed, position and tangential speed have harmonic variations that correspond to the second harmonic of the rotating speed.

Actuation in the polar domain also has several advantages as it introduces targeted action on stiffness and damping. In this work, the controller was developed in the polar coordinate system, while measurements and actuation were still performed in the Cartesian coordinate system. This was due to the fact that the technology used was designed to operate in one direction.

The physical quantities introduced by polar transformation enabled the controller to distinguish the disturbance produced by the unbalance from the transient disturbance exciting the rotor radially. Indeed, when the rotor was subjected only to unbalance excitations, the rotor orbit was circular and the radius of the whirl orbit was constant or changed slowly during run up. The radial velocity was almost nil and the variations were only due to the measurement noise.

Four trapezoidal membership functions were utilized (Fig. 6). The rules selected were:

1) For steady-state behavior, when tangential velocity was high tangential damping was increased by $50 \%$.

2) For transient behavior, when radial velocity was positive, radial stiffness was increased by $50 \%$.

3) If the velocity variation was small or negative, there was no action.

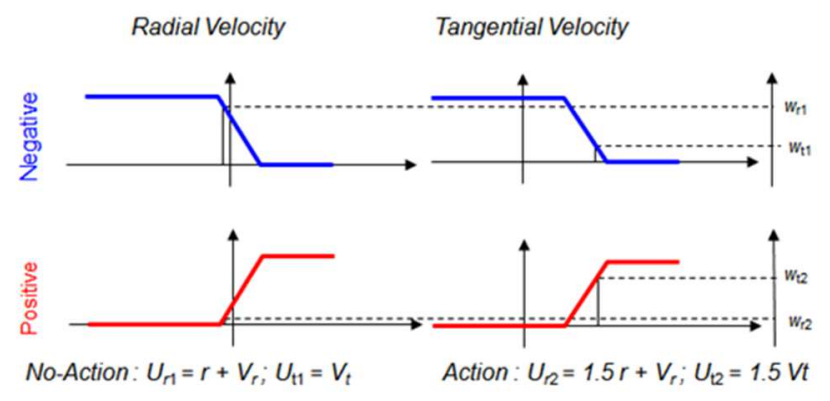

Fig. 6 Membership functions of the polar fuzzy controller

then the fuzzy controller commands were expressed as actions along directions $x$ and $y$ :

$$
\left\{\begin{array}{l}
F_{x}=F_{r} \cos \theta-F_{t} \sin \theta \\
F_{y}=F_{r} \sin \theta+F_{t} \cos \theta
\end{array}\right.
$$

\section{Numerical results}

Numerical simulations were necessary in order to adjust the different parameters of the controllers developed. The fuzzy controllers were compared with the developed SPID. The unbalance responses during run-up from 0 to $10,000 \mathrm{rpm}$ in 100 seconds was assessed. The unbalance distribution used for the numerical simulations was identified experimentally during the experimental study into three planes (Table 2): near drive end bearing (node \#14), at the middle (node \#24) and near non drive end bearing (node \#33). 
Table 2: Unbalance distribution

\begin{tabular}{ccc}
\hline \hline Positions & Amplitude (g. mm) & Phase (degrees) \\
\hline Drive end & 11.4 & 16 \\
Middle & 3.3 & 120 \\
Non-drive end & 10.3 & 62 \\
\hline
\end{tabular}

The displacements in the two directions $\mathrm{X}$ and $\mathrm{Y}$ of the drive end and non-drive end bearings are presented in figure 7. The rms value of the displacement was chosen. The three controllers provided excellent performances, with better behavior for the fuzzy controllers. The two rigid body modes and the first flexible one could be observed when using the SPID controller, while only the first rigid body mode is still observable when using the fuzzy controllers, and it can be noticed that those controllers provide stiffness that makes the bearings more rigid. For the numerical simulations, only the system performances were assessed. The current general shapes and trends are similar to those of the displacement.
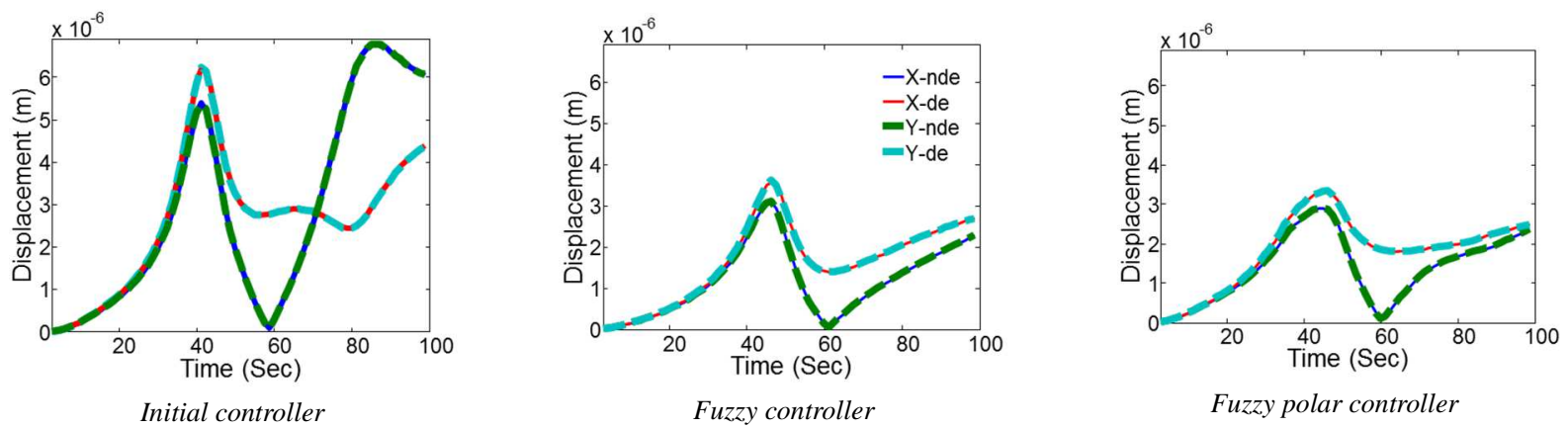

Fig. 7 Unbalance responses during run-up, numerical simulation

\section{Experimental results}

The fuzzy controllers were compared with the initial augmented PID controller delivered with the test rig. The unbalance responses during run-up from 0 to 10,000rpm in 100 seconds was assessed. The results are presented in the two directions of the drive end bearing (V13 \& W13) and the non-drive end (V24 \& W24).
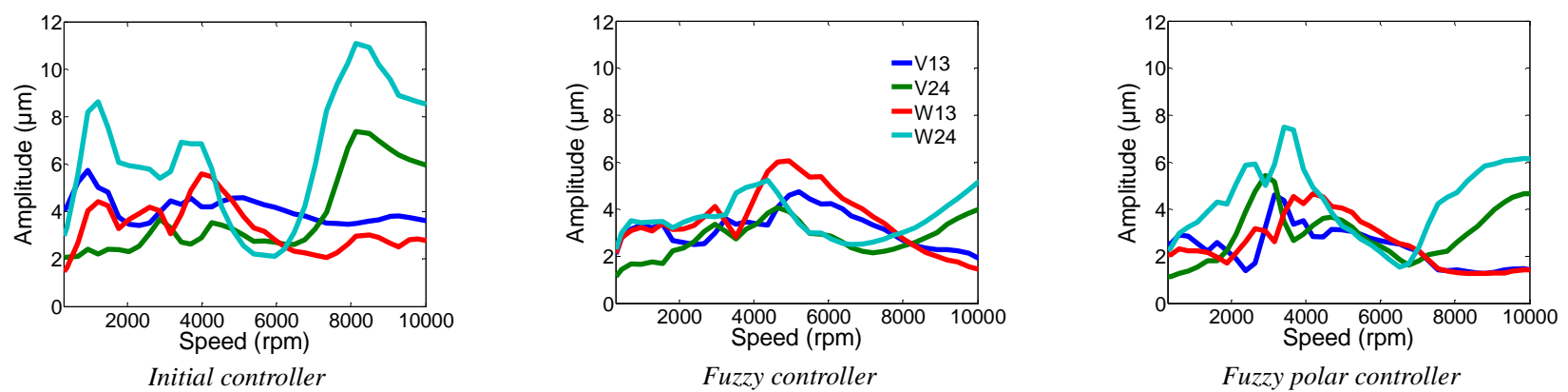

Fig. 8 Unbalance responses during run-up, experimentation

First, the displacement levels resulting from the three controllers assessed are compared in figure 8 . The rms value of the displacement was chosen. The unbalance responses exhibited three critical speeds: the first around 1000rpm, which occurred only when using the initial PID controller, was identified as the foundation mode; the second, in the vicinity of 4000rpm, corresponded to the cylindrical mode; and the last, close to 9000rpm, was identified as the first flexible critical speed. The conical mode was damped too strongly to appear in the unbalance response. The differences observed between experimental and numerical results are due to the uncertainties due to several reasons: differences between assumed and real unbalance distribution, dry friction in test rig shaft (three parts bolted together) and linearization performed in simulation. Uncertainties were not considered in the model. 

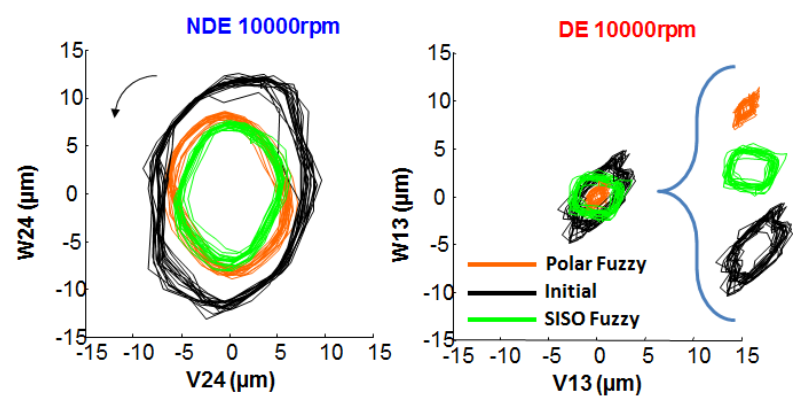

Fig. 9 Rotor orbit at 10,000rpm

The rotor orbits measured at the NDE bearing for the stabilized speed of 10,000 rpm are presented in Fig. 9. The three controllers provided performances that matched the required specifications. From the standpoint of performance, the fuzzy controller presented the best results. Nevertheless, this controller exhibited anisotropic behavior that could be undesirable. This anisotropy was due to the fact that the membership functions were expressed in the Cartesian coordinate system. Consequently, the control forces generated led to a square orbit shape when reaching displacement thresholds.
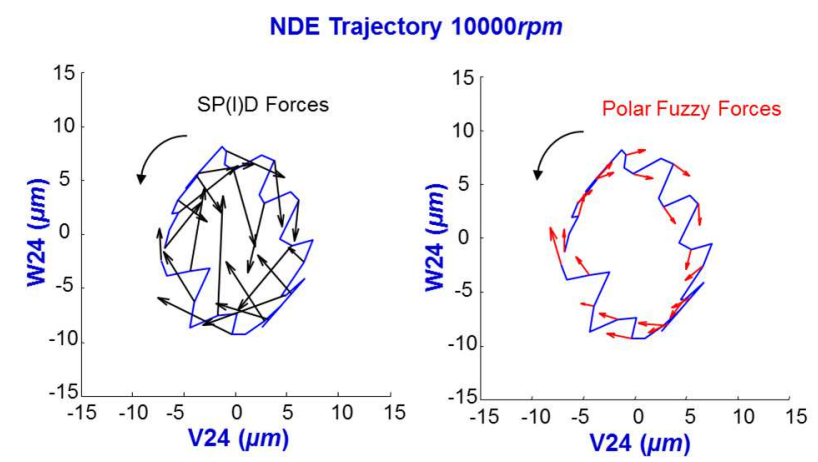

Fig. 10 Control forces, non-drive end bearing at 10,000rpm

The forces applied by the fuzzy polar controller at 10,000rpm are presented in Figure 10. The first plot presents the proportional and derivative forces computed by the SPID. For the sake of clarity, the integral forces are removed because they introduced a constant force in the vertical direction, which counteracts the weights. It can be noticed that the amplitude and the direction of the forces change constantly and the controller is sensitive to the measurement noise. On the other hand, the force applied by the fuzzy controller has almost constant amplitude and a same tangential direction, the utilization of polar quantities with fuzzy logic permits to apply targeted forces.
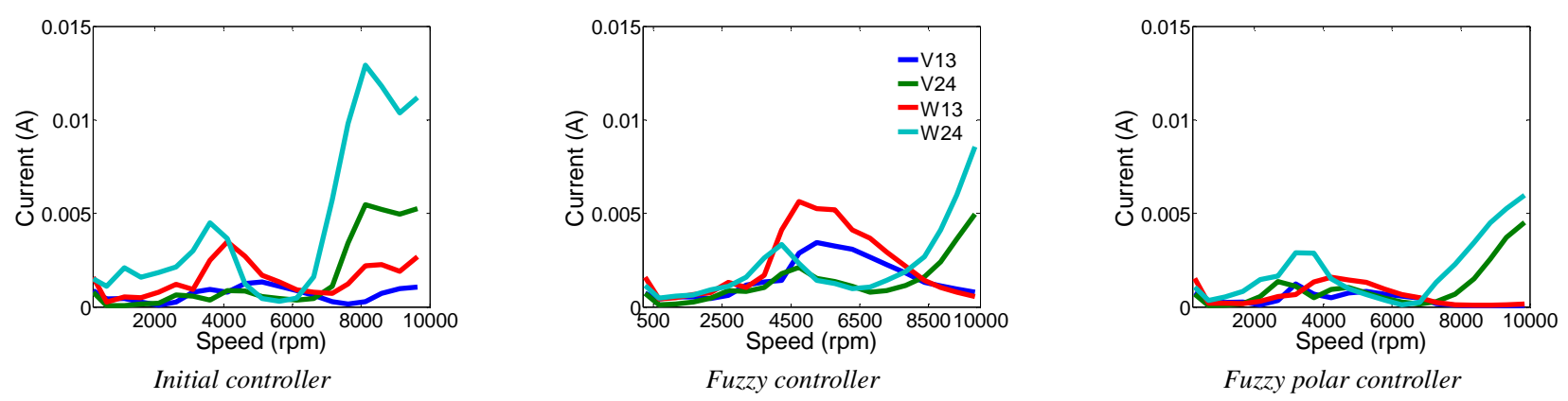

Fig. 11 Unbalance responses during run-up

The rms value of the current was calculated (Fig. 11). It was representative of the energy necessary for the control in each case. At this stage, no calculations were performed to quantify the energy. We estimated that the rms value represented the energy consumed correctly.

The aim was not to minimize the energy necessary for the control, which was negligible with respect to the energy necessary to perform the run-up of the system. However, as AMBs are designed (even partially) as a function of the max 
current, we assumed that the controller using minimum energy could lead to reducing the size of the control cabinet and increasing the margins of power amplifier capacities. The results obtained showed that the fuzzy polar controller presented optimal behavior performance / consumption.

\section{Conclusion}

The mechanical performances and the energy necessary for the control of an academic test rig were assessed numerically and experimentally. The results obtained showed that the model describes accurately the dynamic behavior of the system studied. The differences observed are due to the uncertainties (geometric and environment) that were not considered in the model. The aim was to evaluate the effectiveness of the controllers developed and the possibility of optimizing the energy consumption in order to reduce the size of the control cabinet and increase margins as a function of power amplifier capacity.

Two fuzzy controllers were presented and compared with a PID controller. The first controller was a SISO fuzzy controller which exhibited high performance, with stable and robust behavior. Its weaknesses were anisotropic behavior that led to alternative bending (among other effects) and a permanent nonlinear response. The use of polar coordinates overcame the disadvantages of the SISO fuzzy controller. On the other hand, the polar fuzzy controller provided several gains that must be mastered in order to manage the system's performance, stability and the robustness. Globally, better performances were obtained with fuzzy controllers. Energy consumption was also compared. It was observed that the Fuzzy controllers consumed significantly less energy.

The aim was to select a controller with optimized energy consumption / mechanical behavior, in order to optimize AMB design. The results obtained are encouraging for ongoing development in this area.

The fuzzy controllers were designed to control relatively small disturbances which concern nominal behavior (class A ISO 14839) whereas the fuzzy rules were not adapted for large displacements. Therefore new rules are necessary to improve performances. Also, only these three controllers were assessed. Indeed, for the study to be complete more controllers have to be assessed, but at this stage the aim was to evaluate fuzzy logic based strategies to control turbomachines.

\section{Acknowledgments}

This work is a part of Benjamin DEFOY PhD thesis performed with the support of CIFRE funding $\mathrm{N}^{\circ} 2009 / 0879$. The authors are grateful to the ANRT National Agency for supporting this research.

\section{References}

API 617, Axial and centrifugal compressors and expander-compressors for petroleum, chemical and gas industry service, 7th ed. American Petroleum Institute standard (2002).

Borne, P., Rozinoer, J., Dieulot J.-Y. \& Dubois L., Introduction à la commande floue (1998), p.102, Edition Technip (in French).

Chen, K., Tung, P., Tsai, M. \& Fan Y., A self-tuning fuzzy PID-type controller design for unbalance compensation in an active magnetic bearing, Expert Syst. Appl., (2009). pp. 8560-8570.

Couzon, P.-Y. and Hagopian, J. Der, Neuro-fuzzy active control of rotor suspended on active magnetic bearing, J. Vib. Control, 13(4) (2007), pp. 365-384.

Defoy, B, Alban, T. and Mahfoud, J., Assessment of the effectiveness of a polar fuzzy approach for the control of centrifugal compressors, ASME Journal of Dynamic Systems, Measurement, and Control, vol. 136 (2014), 041004$1-041004-8$.

Fittro, R. L. and Knospe, C. R., The $\mu$ Approach to Control of Active Magnetic Bearings, J. Eng. Gas Turbines Power, Vol 124, No. 3 (2002), pp. 566-570.

Font, S., Duc, G. \& Carrere, F., Commande fréquentielle robuste - Application aux paliers magnétiques, Techniques de l'ingénieur, Mesures Analyses R 7432 (1997) (in French).

Fuh, C.-C. \& Tung, P.-C., Robust stability analysis of fuzzy control systems, Elsevier Science B.V. Fuzzy Sets and Systems, Vol. 88, Issue 3 (1997), pp. 289-298. 
Golob, M. \& Tovornik, B., Modeling and control of a magnetic suspension system, Elsevier Ltd. ISA Trans., Vol. 42, Issue 1 (2003), pp. 89-100.

ISO 14839-1, Mechanical Vibration - Vibration of rotating machinery equipped with active magnetic bearings - Part 1 : Vocabulary (2002).

ISO 14839-2, Mechanical Vibration - Vibration of rotating machinery equipped with active magnetic bearings - Part 2: Evaluation of vibration (2004).

ISO 14839-3, Mechanical Vibration - Vibration of rotating machinery equipped with active magnetic bearings - Part 3: Evaluation of stability margin (2006).

Lalanne, M. and Ferraris, G., Rotordynamics Prediction in Engineering (1998), p.252, 2nd Edition, John Wiley \& Sons.

Lei, S., and Palazzolo, A. B., Control of flexible rotor systems with active magnetic bearings, Journal of Sound and Vibration, Vol. 314, Issues 1-2 (2008), pp.19-38.

Mahfoud, J. \& Der Hagopian, J., Fuzzy Active Control Of Flexible Structures By Using Electromagnetic Actuators, ASCE's Journal of Aerospace Engineering, Vol. 24, No. 3 (2011), pp. 329-337.

Mahlis, M., Gaudiller, L. \& Der Hagopian, J., Fuzzy Modal Active Control of the Dynamic Behavior of Flexible Structures, Journal of Vibration and Control, 11 (2005), DOI: 10.1177/10775463045046028.

Maslen, E. H., Smart Machine Advances in Rotating Machinery, Proceeding 9th International Conference on Vibrations in Rotating Machinery (2008), IMechE, Exeter, UK, pp.3-14.I.

Matlab: The MathWorks - Matlab and Simulink for Technical Computing, www.mathworks.com.

Qiao, W. Z. \& Mizumoto, M., PID type fuzzy controller and parameters adaptive method, Fuzzy Sets and Systems, vol. 78 (1996), pp. 23-35.

Sahinkaya, N. M., Abulrub, A.-H. G., Burrows, C. R. and Keogh, P. S., A Multiobjective Adaptive Controller for Magnetic Bearing Systems, J. Eng. Gas Turbines Power, 132 (2010), pp.122503.

Schweitzer, G. and Maslen, E. H, Magnetic Bearings, Theory, Design, and Application to Rotating Machinery (2009), Springer-Verlag, p.535.

Swann, M. K. A., Sarichev, P. and Tsunoda, E., A diffusion model for active magnetic bearing systems in large turbomachinery, Proceeding 11th ISMB (2008), Japan, pp. 380-384. 